\title{
Frequency scans and resonance mode analysis for resonance problems identification in power networks in presence of harmonic pollution
}

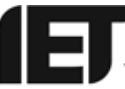

ISSN $2515-0855$ doi: 10.1049/oap-cired.2017.0807 www.ietdl.org

\author{
Loïc Eggenschwiler ${ }^{1} \bowtie$, Olga Galland ${ }^{1}$, Damien Chollet ${ }^{2}$, \\ Fabrice Decorvet ${ }^{3}$, Dominique Roggo ${ }^{4}$, Patrick Favre-Perrod ${ }^{1}$ \\ ${ }^{1}$ Energy Institute, HES-SO Fribourg, Fribourg, Switzerland \\ ${ }^{2}$ Services Industriels de Lausanne, Lausanne, Switzerland \\ ${ }^{3}$ Services Industriels de Genève, Geneva, Switzerland \\ ${ }^{4}$ Institute of Systems Engineering, HES-SO Valais, Sion, Switzerland \\ $凶$ E-mail: loic.eggenschwiler@hefr.ch
}

\begin{abstract}
The understanding of harmonics propagation in power systems recently gained interest. In high-voltage (HV) networks, the current trend of undergrounding is modifying the network resonance frequencies. In low-voltage (LV) distribution systems, the increasing deployment of decentralised generation induces new harmonic pollution, which could interfere with power line communications. In this analysis, the most important elements of power systems (overhead lines, underground cables, power transformers, linear loads and inverters) are modelled in frequency and integrated into frequency scan and resonance mode analysis methods. Two network types are analysed to point out the specificities of each network level: the first one is an extra high voltage (EHV)/HV network (380, 220, 125 and $50 \mathrm{kV}$ ) and the second one is a part of a medium-voltage/LV distribution system (20 kV and $400 \mathrm{~V})$. With this method, the network behaviour in a large frequency bandwidth (up to $5 \mathrm{kHz}$ for transport grids and up to $200 \mathrm{kHz}$ for the distribution system) can be predicted. Recent network impedance measurements contribute to the validation of this method by showing the same trends and resonance frequencies close to the ones visible in the simulation results.
\end{abstract}

\section{Introduction}

With the increased use of power electronics, distribution system operators (DSOs) are facing the increasing significance of a known issue: harmonic pollution. Due to its switching nature, each power electronic converter injects harmonics into the network. In high-voltage (HV) power systems, harmonics, e.g. are produced by flexible AC transmission systems (FACTS) and high-voltage direct current converters as well as magnetic components. The combination of harmonics and resonances at the same frequencies can lead to equipment damages due to overvoltages, losses and interferences with communication systems [1]. In low-voltage (LV) distribution systems, decentralised generation (DG) could imply interferences between produced harmonics and power line communications (PLC) [2]. All these increasing risks contribute to the DSOs interest in this research field. Indeed, harmonic sources are now well identified, but for some DSOs, the difficulties in measuring the harmonics' propagation in networks imply that the link between these harmonics, network resonances and their potential effects is uncertain in many practical cases. Furthermore, the coupling of network levels with transformers permits resonances to 'propagate' from one network level to another [3], and this phenomenon increases the resonance analysis study's complexity.

In the transmission system, e.g. resonances have already been experienced. Active filters have been considered as a mitigation measure. The consequence of this problem was malfunctioning of the ripple control used for public lighting. In HV sub-transmission systems, the trend to increasingly undergrounded (UG) networks is expected to change the resonance frequencies since UG cables are more capacitive than overhead $(\mathrm{OH})$ lines [3]. In distribution systems, smart grid devices like smart meters, which use PLC to communicate, are more prone to interference in cases where there is a high penetration of decentralised generation. Hence, methods and tools to simulate and predict the network harmonic behaviour are needed at every network voltage level. The method used here to analyse the network is composed of the three following steps:

- First, all the influent network elements are modelled in the frequency domain.

- Then the network admittance matrix, which takes the studied network topology into account, is built for each frequency of the band of interest.

- Finally, the frequency scan method [4] is used to identify resonances (at which node and around which frequency) and the resonance mode analysis (RMA) [5-7] gives the information about resonances origins.

In this paper, two typical networks are analysed in the frequency domain, one representing the HV sub-transmission networks and the other one illustrating the MV and LV distribution networks. Both these typical networks are described in Section 2. The used elements models are briefly explained in Section 3, and the analysis methods applied to these models are discussed in Section 4. The simulation results are discussed in Section 5 and the applicability of the analysis method is discussed in Section 6 .

\section{Applications}

\subsection{HV sub-transmission system}

The first network analysed here is the typical HV sub-transmission system of Services industriels de Lausanne (SIL). This Swiss DSO plans to change its current operational topology shown in Fig. 1 to 


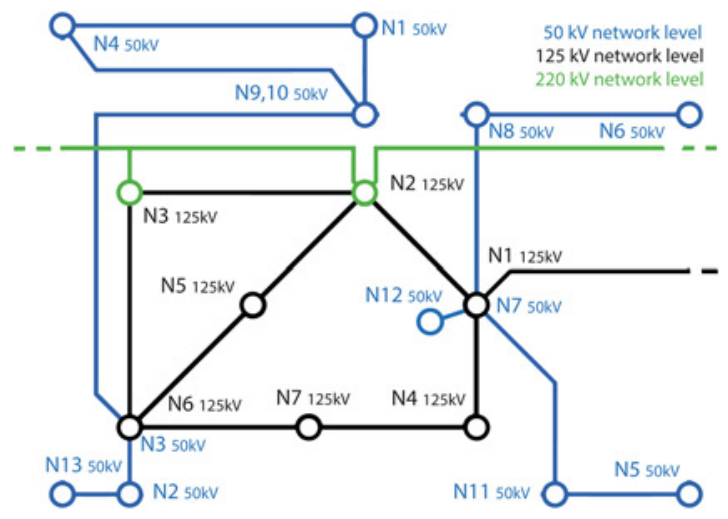

Fig. 1 SIL current operational topology

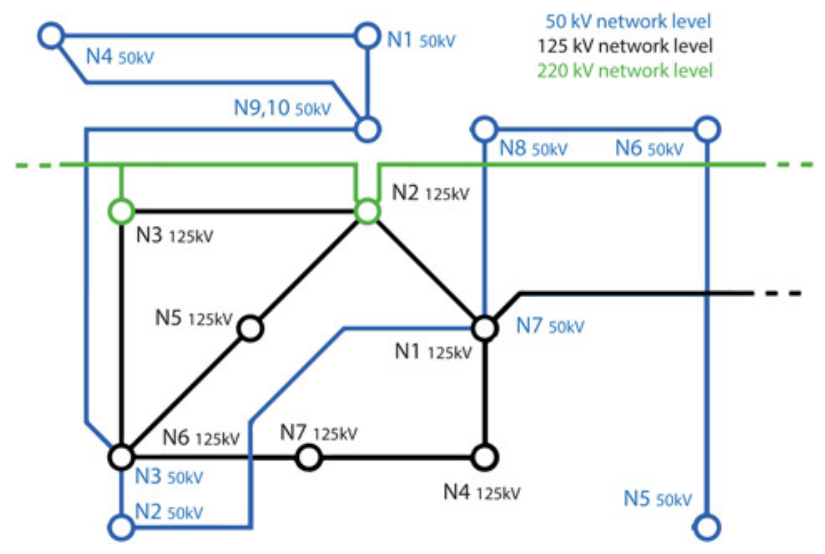

Fig. 2 SIL future operational topology

Table 1 SIL study considered system boundaries

\begin{tabular}{lccc}
\hline Description & Voltage, kV & Nodes & Lines \\
\hline transmission system & 380 & 33 & 49 \\
18 transformers & $380 / 220$ & & \\
& 220 & 131 & 196 \\
eight transformers & $220 / 125$ & & \\
sub-transmission system & 125 & 42 & 60 \\
four transformers & $125 / 50$ & 13 & 16 \\
& 50 & & \\
\hline
\end{tabular}

the one of Fig. 2 in the future, essentially by increasing its undergrounding.

This change of topology could imply some modifications on its harmonic behaviour. If the frequency of one of its resonances exactly matches a harmonic current injected by an existing power electronic device, the resulting interaction could cause damages in the network or cause outages. The same phenomenon could happen if one of these resonances occurs at the carrier frequency of the centralised control.

The network impedance model in the frequency domain gives useful information about which frequencies are sensitive and at which any current injection must be absolutely avoided. The upstream network levels have also been considered and modelled in this study. The system boundaries are placed on the Swiss part of the power system, as shown in Table 1.

\section{$2.2 \quad L V$ distribution system}

The frequency scan method used in this work has also been applied to a representative distribution system of another DSO, SIG. The area of interest, exposed in Fig. 3, is limited to the MV lines

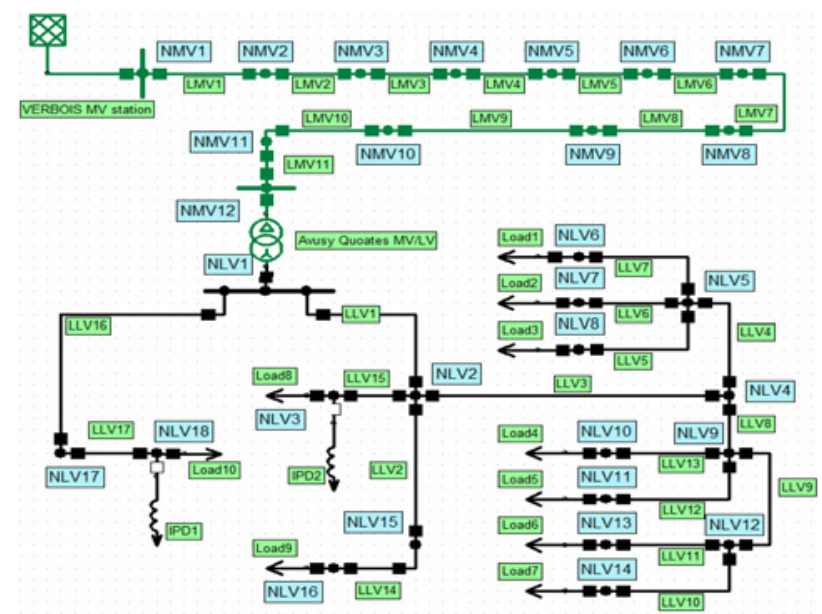

Fig. 3 SIG distribution network

(from the HV/MV transformation station), the MV/LV transformation station, as well as two of the eight transformer departures. The MV part $(18 \mathrm{kV})$ is composed of $\mathrm{OH}$ lines and UG cables and the LV part $(400 \mathrm{~V})$ is only built with UG cables. The MV/LV transformer is a Dyn oil-type transformer with a rated power of $630 \mathrm{kVA}$. Ten linear loads are modelled to emulate the houses behaviour and influence on the network impedance.

This distribution network has been chosen as the object of this study because its DG penetration is high and because low data transfer rates in the PLC were observed between the smart grid equipment installed at MV/LV station and at the coupling point of DG. Since this equipment uses frequencies of 63 and $73 \mathrm{kHz}$ for its communication carrier signal and previous measurements had shown high harmonic currents (THD $>500 \%$ ) up to $70 \mathrm{kHz}$, the cause of the low rate of transmitted data could be interferences with the harmonic pollution. A better knowledge of the network impedances is then required to understand these phenomena.

\section{$3 \quad$ Modelling}

\section{1 $\mathrm{HV} \mathrm{OH}$ lines and UG cables}

HV power lines are modelled on the basis of their physical and geometrical characteristics, using the models proposed in [8]. These models are used for $\mathrm{OH}$ lines and UG cables with rated voltages of $20,125,220$ and $380 \mathrm{kV}$. The geometrical parameters required to model these elements are exposed in Figs. 4 and 5 for $\mathrm{UG}$ cables and $\mathrm{OH}$ lines, respectively.

\subsection{UG cables}

A significant part of the LV lines is UG and thus only the UG power cable models are needed in this work. The typical geometry of LV power cables is shown in Fig. 6.

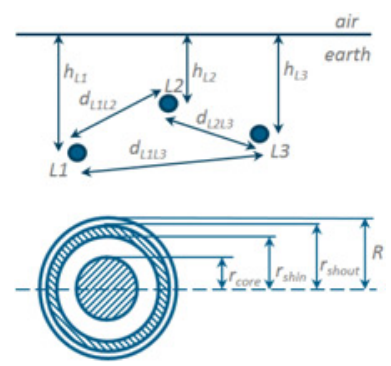

Fig. 4 HV power cables geometry 


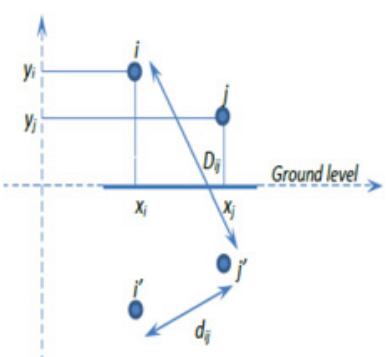

Fig. 5 HV OH lines geometry

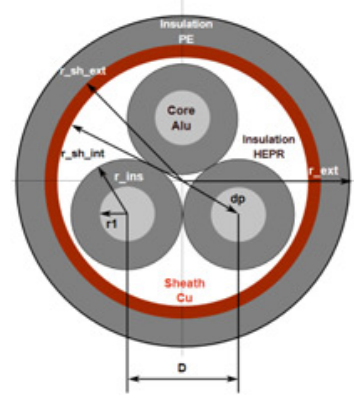

Fig. 6 LV power cables geometry

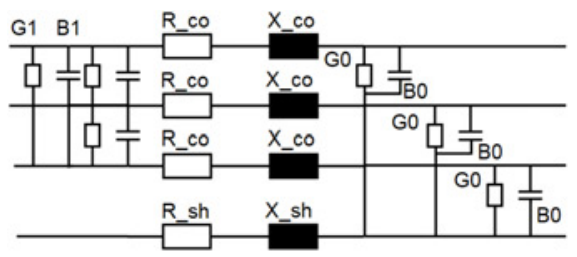

Fig. 7 LV power cable resulting model

The resulting model, with all the considered phenomena, is exposed in Fig. 7.

The core inductance $X_{\mathrm{co}}$, the core resistance $R_{\mathrm{co}}$ (considering the skin effect), as well as the core-to-core capacity $C_{1}$, are determined using formulae developed in [9]. To calculate the core-to-sheath capacity $C_{0}$, a well-known formula is given in [10]. It is used to determine the conductance $G_{0}$ and the susceptance $B_{0}$. The sheath impedance $\left(R_{\mathrm{sh}}\right.$ and $\left.X_{\mathrm{sh}}\right)$ depends only on the sheath internal and external radius $r_{\mathrm{sh} \text {,in }}$ and $r_{\mathrm{sh} \text { out }}$ [11]. When all these elements are determined, the same method as for HV power cables [8] is used to integrate $\mathrm{LV}$ power cables in an admittance matrix form.

\subsection{Power transformers}

The inductive equivalent model, widely used and accurate enough to emulate transformers behaviour at the industrial frequency $(50 \mathrm{~Hz} /$ $60 \mathrm{~Hz}$ ), is not suitable when the analysis frequency bandwidth is increased up to a few kilohertz. In distribution systems, studies focussed on PLC interactions have to consider frequencies in the european committee for electrotechnical standardization (CENELEC) band A (up to $95 \mathrm{kHz}$ ). At such frequencies, stray capacitances of the transformer have a significant effect. The grey-box transformer model gives a good estimation of a specific transformer's behaviour, without the need to measure it. Each winding, as well as the magnetising branch, is represented by RLC circuits. A capacitive impedance is considered between both the sides and between the HV side and the earth. The lumped equivalent circuit from [12] is shown in Fig. 8.

To obtain a three-phase transformer model, three lumped equivalent circuits are connected according to the transformer coupling (YNyn, Dyn, YNd, ...).

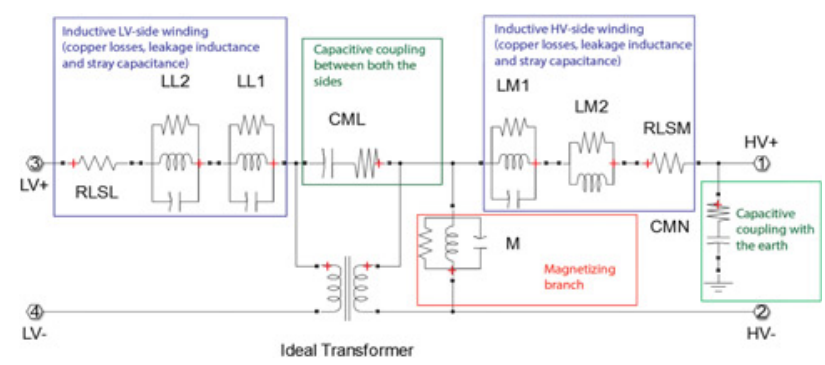

Fig. 8 Lumped equivalent circuit of the transformer grey-box model used in the frequency domain

\subsection{Distribution loads}

Three possible load models have been used in this work: series RL, parallel RL and the international council on large electric systems (CIGRE) model [13]. These models, exposed in Fig. 9, can be expressed depending on their rated voltage $V$, the active and reactive power $P$ and $Q$, and the harmonic order $h$

$$
\begin{aligned}
& R=P \frac{V^{2}}{P^{2}+Q^{2}}, \quad R=\frac{V^{2}}{P}, \quad R_{\mathrm{s}}=\frac{V^{2}}{P}, \quad X_{\mathrm{s}}=h \times 0.073 R_{\mathrm{s}} \\
& X=h Q \frac{V^{2}}{P^{2}+Q^{2}}, \quad X=h \frac{V^{2}}{Q}, \quad X_{\mathrm{p}}=\frac{h R_{\mathrm{s}}}{6.7(Q / P)-0.74}
\end{aligned}
$$

\subsection{Power electronics}

Classical power electronic devices, such as standard inverters used to inject DG into the power system, can be modelled as a current source with a shunt admittance. This method permits to evaluate the influence of its admittance on the network and the harmonic pollution injected by a given type of inverter. In the considered equivalent network exposed in Fig. 10, the current source $i_{\mathrm{INV}}$ is the desired source at industrial frequency $(50$ or $60 \mathrm{~Hz})$ and another current source $i_{\text {Harm }}$ represents the injected harmonic pollution. An existing method [14] to consider the inverter control loop in the shunt admittance $Y_{\text {INV }}$ determination has been used here.
Series $R L$

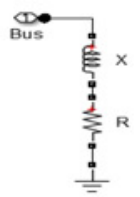

Parallel RL

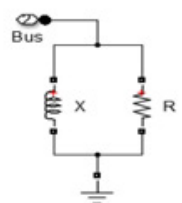

\section{CIGRE model}

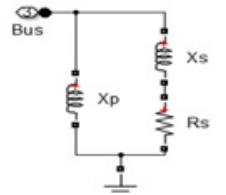

Fig. 9 Three possible linear load models

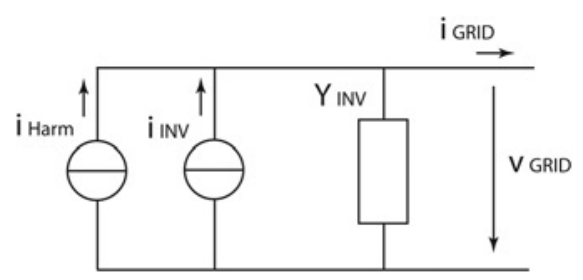

Fig. 10 Equivalent circuit of a $D G$ inverter 


\section{Methods}

As stated above, based on its geometry each network's component admittance is determined and the admittance matrix $Y_{\mathrm{BUS}}$ is built for each frequency inside the bandwidth of interest.

\subsection{Frequency scan}

The frequency scan yields the nodal frequency response. In other words, this is the nodal impedance equivalent to the corresponding nodal impedance matrix diagonal element [4].

\subsection{Resonance mode analysis}

The RMA, based on Eigen analysis, unlike the frequency scan determines impedances in the modal coordinate system. The nodal impedance matrix transformation into the modal impedance matrix is described in [5-7].

This method allows us to analyse the global network resonance response by indicating the network critical mode at each studied frequency. The RMA provides valuable information on participation factors (PF) at each critical mode. Using PF, the nodes contribution to a given resonance mode can be obtained and the resonance 'origin' can be identified. The RMA permits to illustrate changes in resonance behaviour of several network configurations or/and expansion networks.

\section{Simulation results}

\subsection{HV sub-transmission system of SIL}

The frequency scan as well as the RMA of this sub-transmission system have been analysed. The results of the RMA, also called critical modes, represent the network global resonant behaviour. The comparison between the critical mode of the current and the future operational topologies, exposed in Fig. 11, highlights the frequencies where there is a significant difference, located around $1500 \mathrm{~Hz}$.

First, these results show that the changes in the network resonance, implied by the changes of topology, will not affect the centralised ripple control (at $475 \mathrm{~Hz}$ ) in the future. Secondly, most of the nodes with the highest contribution to the resonant behaviour are identified for each resonance of the critical mode. When the $50 \mathrm{kV}$ level only is analysed, six resonance peaks are found on the frequency scan. The five highest PF of each peak are reported in Table 2. These PF inform about which nodes are 'the most responsible' of a resonance peak. The only peak influenced by nodes in the $50 \mathrm{kV}$ level is the resonance peak 5 (around $1519 \mathrm{~Hz}$ ). It is therefore coherent that the resonant behaviour is only influenced around this frequency when a change of topology happens in the $50 \mathrm{kV}$ network level.

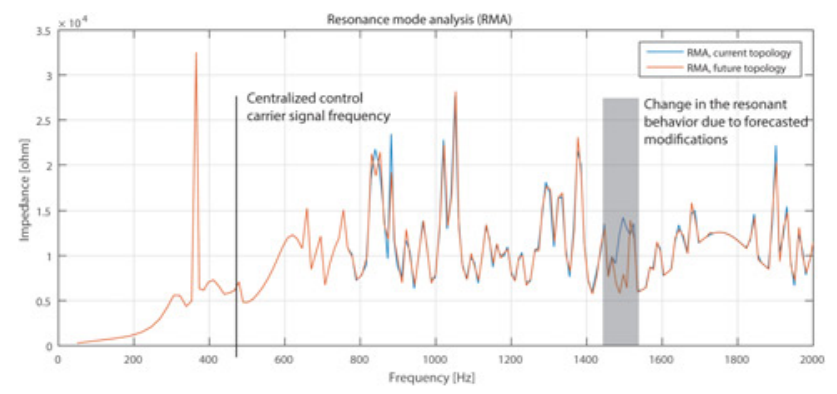

Fig. 11 SIL changes influence on the network critical mode
Table 2 Five most important participation factors (PF) of the six located resonance peaks

\begin{tabular}{|c|c|c|c|c|c|}
\hline \multicolumn{2}{|c|}{ Peak 1, @ $1294 \mathrm{~Hz}$} & \multicolumn{2}{|c|}{ Peak 2, @ 1333 Hz } & \multicolumn{2}{|c|}{ Peak 3, @ $1371 \mathrm{~Hz}$} \\
\hline \multicolumn{2}{|c|}{ Level: 220} & \multicolumn{2}{|c|}{ Level: 220} & \multicolumn{2}{|c|}{ Level: 220} \\
\hline Node & PF & Node & PF & Node & PF \\
\hline 81 & 0.07 & 87 & 0.31 & 21 & 0.14 \\
\hline 42 & 0.05 & 113 & 0.09 & 63 & 0.12 \\
\hline 100 & 0.05 & 114 & 0.07 & 101 & 0.03 \\
\hline \multicolumn{2}{|c|}{$\begin{array}{l}\text { Peak 4, @ } 1445 \text { Hz } \\
\text { Level: } 220\end{array}$} & \multicolumn{2}{|c|}{$\begin{array}{c}\text { Peak 5, @ } 1519 \mathrm{~Hz} \\
\text { Level: } 220\end{array}$} & \multicolumn{2}{|c|}{$\begin{array}{l}\text { Peak 6, @ } 1593 \mathrm{~Hz} \\
\text { Level: } 220\end{array}$} \\
\hline Node & PF & Node & PF & Node & PF \\
\hline 110 & 0.12 & 7 & 0.09 & 81 & 0.09 \\
\hline 36 & 0.02 & 6 & 0.09 & 42 & 0.05 \\
\hline 47 & 0.02 & 9 & 0.09 & 100 & 0.05 \\
\hline
\end{tabular}

\subsection{LV distribution system of SIG}

The simulation results of the studied typical distribution network area illustrate two interesting phenomena: the adding of an inverter tends to induce a new resonance, and this new resonance is visible at frequencies lower than its switching frequency at $15 \mathrm{kHz}$ (cf. Fig. 12).

In a related project, a measurement device is developed with a large frequency bandwidth $(2-200 \mathrm{kHz})$. This device, described in [15], was used to measure the impedance of the same real network. The comparison between these measurements and the simulation results are shown in Fig. 13 for two important nodes of the network: the first one is on the LV side of the distribution transformer and the second one is $42 \mathrm{~m}$ further, in a house where smart meters using PLC and PV inverters are located.

The shape of both measured and calculated curves is similar. The first cause of difference could be the boundaries of the modelled system (measurements are done on all the system) and the second cause could be the measurement device range of accuracy. The causes of these differences include measurement errors and modelling inaccuracies. Influence of unknown loads and sources connected to the same LV feeder have not been considered in the simulation model. This equipment could be the source for the

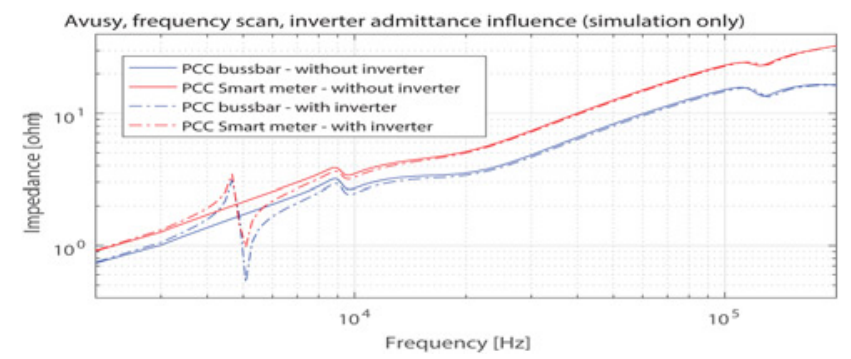

Fig. 12 Inverter influence on the network impedance



Fig. 13 Total network impedance measurements 
impedance variations measured in the range $15-40 \mathrm{kHz}$. However, the network resonances are visible for both measurements and simulation results in the same range of frequencies.

\section{Conclusion}

Network resonances have been identified in systems by both measurement and simulation. Issues implied by the integration of renewable energy production are highlighted: on one hand, each inverter introduces harmonic currents and on the other hand, this inverter could induce resonances in the network impedance. Based on recent experience, it is important to understand these effects in order to maintain the functionality of PLC and ripple control systems. The modelling framework summarised and applied in this paper will help the DSOs to do so early in the planning process.

\section{References}

1 Temma, K., Ishiguro, F., Toki, N., et al.: 'Clarification and measurements of high frequency harmonic resonance by a voltage sourced converter', IEEE Trans. Power Deliv., 2005, 20, pp. 450-457

2 Larsson, E.O.A., Bollen, M.H.J., Wahlberg, M.G., et al.: 'Measurements of high-frequency $(2-150 \mathrm{kHz})$ distortion in low-voltage networks', IEEE Trans. Power Deliv., 2010, 25, pp. 1749-1757
3 Galland, O., Leu, D., Berner, V., et al.: 'Resonance analysis of a transmission power system and possible consequences of its undergrounding', DEMSEE'15, 2015

4 Das, J.C.: 'Power system analysis: short-circuit load flow and harmonics' (CRC Press, Taylor \& Francis Group, New York, 2012, 2nd edn.)

$5 \mathrm{Xu}$, W., Huang, Z., Cui, Y., et al.: 'Harmonic resonance mode analysis', IEEE Trans. Power Deliv., 2005, 20, pp. 1182-1190

6 Cui, Y., Xu, W.: 'Harmonic resonance mode analysis using real symmetrical nodal matrices', IEEE Trans. Power Deliv., 2007, 22, pp. 1989-1990

7 Cui, Y., Wang, X.: 'Modal frequency sensitivity for power system harmonic resonance analysis', IEEE Trans. Power Deliv., 2012, 27, pp. 1010-1017

8 Rivas, R.A.: 'Section 9: overhead transmission lines and underground cables', in 'Handbook of electric power calculations' (The McGraw-Hill Companies, New York, 2001, 3rd edn.), pp. 9.1-9.33

9 Idir, N., Weens, Y., Franchaud, J.J. 'Skin effect and dielectric loss models of power cables', IEEE Trans. Dielectr. Electr. Insul., 2009, 16, pp. 147-154

10 Küpfmüller, K., Mathis, W., Reibiger, A.: 'Theoretische Elektrotechnik' (Springer-Verlag Berlin Heidelberg, Berlin, 2005, 16th edn.)

11 Andrieu, C., Dauphant, E., Boss, D.: 'A frequency-dependent model for a MV/LV transformer'. Int. Conf. on Power Systems Transients, Budapest, Hungary, 1999

12 Kikkert, C.J.: 'A PLC frequency model of 3 phase power distribution transformers'. SmartGridComm, Int. Conf. on, Tainan, 2012, pp. 205-210

13 Eggenschwiler, L., Galland, O., Favre-Perrod, P., et al.: 'Impact of aggregate linea load modeling on harmonic analysis: a comparison of common practice and analytical models', IEEE Trans. Power Deliv., 2003, 18, pp. 625-630

14 Wasterlain, S., Parisod, H., Roggo, D., et al.: 'EMC studies on grid integration of distributed energy resources. A $10 \mathrm{~kW}$, IEC-61850 compliant inverter design and preliminary test results', SPEEDAM, 2016, pp. 1059-1066

15 Roggo, D., Merendaz, L., Furrer, D.: 'Online 2 to $150 \mathrm{kHz}$ grid impedance meter' CIRED 2013, Stockholm, 2013, pp. 1-4 